Polish Journal of Microbiology

2016, Vol. 65, No 4, 391-398

MINIREVIEW

\title{
Viral Infection of the Heart: Pathogenesis and Diagnosis
}

\author{
MATEUSZ KUFFNER ${ }^{1,2}$, AGNIESZKA PAWLAK ${ }^{1,2}$ and MACIEJ PRZYBYLSKI ${ }^{3 *}$ \\ ${ }^{1}$ Department of Invasive Cardiology, Central Clinical Hospital of the Ministry of Interior, Warsaw, Poland \\ ${ }^{2}$ Mossakowski Medical Research Centre Polish Academy of Sciences, Warsaw, Poland \\ ${ }^{3}$ Chair and Department of Medical Microbiology, Medical University of Warsaw, Warsaw, Poland
}

Submitted 9 October 2015, revised 27 January 2016, accepted 18 April 2016

Abstract

Viral infections of the heart cause serious clinical problems, either as infectious myocarditis, which usually is a consequence of acute infection or as idiopathic dilated cardiomyopathy, resulting rather from a chronic infection. This minireview presents an up-to-date view on pathomechanisms of viral infection of the heart tissues, the role of immune system in controlling infectious process at its various stages and current possibilities of recognizing viral infection of the heart with use of both cardiological and virological methods. Our goal was to present the variety of known viral agents causing heart infection, level of complexity in mutual virus-cell interactions, and consequent clinical scenarios.

Ke y word s: dilated cardiomyopathy, infectious myocarditis, viral infection of the heart tissue, virus-cell interaction

Viral infection of the heart is an important cause of serious clinical problems: infectious myocarditis (MC) in its various forms (fulminant, acute and chronic) and idiopathic dilated cardiomyopathy (iDCM) are crucial consequences of viral infection (Blauwet and Cooper, 2010). From a classical point of view, the group of cardiomyotropic viruses included, first of all, human enteroviruses (HEV), with major agents of acute $\mathrm{MC}$ : coxsackieviruses A (CVA) and B (CVB). Group of other viruses thought to be responsible for infections of the heart was rather immutable for decades and consisted of rubella virus (RUBV), influenza viruses A and B (FluAV, FluBV), human adenoviruses (HAdV) and paramyxoviruses (mumps virus - $\mathrm{MuV}$, and parainfluenzaviruses - HPIV) (Dennert et al., 2008). Constant improvement in sensitivity of direct virological diagnostic methods, especially introduction of molecular biology techniques, revealed presence of new viral agents in endomyocardial biopsy (EMB) samples, and thus significantly changed the picture of MC etiology. Nowadays, the most prevalent virus found in EMB samples is parvovirus B19 (B19V). "Classical" cardiomyotropic viruses remain on the list, but increasing role of herpesviruses: human herpesvirus 6 (HHV-6), cytomegalovirus (CMV), Epstein-Barr virus (EBV) and herpes simplex viruses (HSV-1 and HSV-2) was also perceived (Kühl and Schultheiss, 2009; Basso et al., 2013).
According to the current model of acute cytopathic viral infection of myocardium, which is seen in enteroviral and adenoviral diseases, a virus reaches the heart with bloodstream from other, usually primary sites of an infection, in the form of a cell-free virion or within infected leukocytes, mainly lymphocytes or macrophages (Kühl and Schultheiss, 2009). Cardiomyocytes are the most important target cells for these viruses. Infection within myocardium often results in acute inflammation, but may also lead to non-inflammatory damage of cells, and as a consequence, to infectionrelated cardiomyopathy. Acute MC is dominated by Th1 and Th17 responses (Huber et al., 2002; Yuan et al., 2010), while activation of Th2 immune reactions is necessary for developing of chronic MC with fibrosis and iDCM (Fairweather et al., 2004; Abston et al., 2012). The main opposition between these two processes (Th1 immune response inhibits Th2 reaction and vice versa), is also complemented by important role of Th17 response, which - being a part of Th1-related inflammatory response - is also believed to induce post-inflammatory cardiac remodeling, characteristic for iDCM (Fairweather et al., 2004). Pathophysiological mechanism of enteroviral and adenoviral MC in humans is still not fully understood, but it is suspected, that this is a three-phase process, as was observed in murine models (Kawai, 1999).

\footnotetext{
* Corresponding author: M. Przybylski, Chair and Department of Medical Microbiology, Medical University of Warsaw, Warsaw, Poland; e-mail: maciej@conexion.pl
} 
Heart infection begins when virus invades the cardiomyocytes with the participation of a specific receptor. Coxsackievirus uses coxsackie-adenovirus receptor (CAR), which is a junctional protein. The same structure is used by HAdV (Kühl et al., 2003). In the absence of CAR expression on cardiomyocytes, the viral invasion of these cells is impossible (Shi et al., 2009). The decay accelerating factor (DAF, CD55) is a co-receptor for HEV internalization, and av-type integrins are needed for adenovirus penetration (Bergelson et al., 1997; Stewart and Nemerow, 2007). As long as this stadium is adequately treated, it may not cause significant damage. It can also result in a heart failure or death due to the direct cytopathic effect caused by an active replication (Herzum et al., 1994).

The first, acute stage of an infection, can end with elimination of the virus from the heart and renovation of damaged tissue. The innate immunity is the first line of defense against virus. This conservative system activates the inflammatory process by tolllike receptors (TLRs), especially TLR-3 and TLR-4, which are located in large quantities in the cells of cardiovascular system. TLR signaling is induced by a variety of ligands which are associated with infectious pathogens (Yajima, 2011). It is interesting, that the disruption of TLR-3 is irrelevant to the interferon beta (IFN- $\beta$ ) mRNA expression in the heart, while TLR-3 deficiency suppresses the expression of class-I interferon regulatory factors (IRF) in the dendritic cells (DCs) infected by CVB (Negishi et al., 2008). This suggests a major role of TLR-3 signaling in response to heart infection, however, its role has not been studied specifically in the cardiomyocytes (Yajima, 2011). The receptors mentioned above recognize foreign antigens, trigger the activation of nuclear transcription factors and lead to the production of inflammatory cytokines (Sagar et al., 2012). TLR effects are mediated by few intracellular pathways, especially myeloid differentiation factor-88 (Myd88) (activated by all TLRs, except TLR-3) and TIR-domain-containing adapter inducing interferon- $\beta /$ TIR-containing adaptor molecule (TRIF/TICAM) (stimulated by TLR-3 and TLR-4). Next step is up-regulation of inflammatory cytokines, by interleukin-associated kinases (IRAK4), TNF receptor-associated factor (TRAF6), TGF- $\beta$-activated kinase 1 (TAK1), nuclear factor kappa-light-chainenhancer (NF- $\kappa \mathrm{B})$, and mitogen-activated protein (MAP) kinases activation. The production of INF- $\beta$ is also regulated by serine/threonine protein kinase (TBK) and interferon regulatory factor 3 (IRF3) (stimulated by TRIF/TICAM) (Lafyatis and Farina, 2012). The activation of non-TLR sensors like retinoid acid inducible gene I (RIG-I), melanoma differentiation associated protein 5 (MDA5) and Laboratory of Genetics and Physiology 2 (LGP2) is another way to induce a immune response. RIG-I like receptors (RLRs) recognize presence of cytoplasmic double stranded RNA and upkeeps the production of IFN- $\beta$. The innate immunity in cooperation with Myd88 adjust inflammation, infiltration and production of cytokines (IL-1, IL-2, IL-6, IL-10, TNF- $\alpha$, IFN- $\gamma$ and C-X-C motif chemokine 10 (CXCL10) (Fuse et al., 2005; Yajima, 2011). They are thought to play a major role in defense against viral infection through the attraction and activation of immune cells. IL- 6 protects the myocardium in the early inflammatory stage, but persistent activation of IL-6 promotes heart injury through disturbance of viral clearance and impaired rising of circulating TNF- $\alpha$ (Tanaka et al., 2001). Endocrine, paracrine and autocrine influence of cytokines on cardiomyocytes also plays big role in fighting the disease. CVB infection is associated with an activation of Janus kinase - signal transducer and activator of transcription (JAK-STAT) signaling pathway in the heart cells (Yasukawa et al., 2003) and this activation is observed at very early stages of the immune response. IFN- $\beta$ is very important for limiting viral presence in the heart. Limited expression or absence of this cytokine causes the increase of mortality. The most important features of intracellular reaction to a viral infection of the heart are depicted in Fig. 1. DCs, natural killer (NK) cells and macrophages migrate to the heart in response to the massive cytokine production induced by viral invasion, and minimalize virus propagation, mostly via direct cytotoxic effect (Yajima, 2011). First, acute phase of the viral invasion of cardiomyocytes, described above, takes only few days. It can be manifested by fever, weakness, rash, muscle pain and joint pain. It can also be accompanied by symptoms of respiratory or gastrointestinal viral infection.

The second stage of the infection is subacute and lasts weeks to months. It is characterized by more sublimated immune reactions. Signals from the innate immunity system contribute also to the activation of specific $\mathrm{T}$ and $\mathrm{B}$ lymphocytes, responding to viral antigens. The highest point of antibodies production also occurs in this phase. Antibodies, which are produced to destroy viruses, often react with the structures of human heart and can cause damage of myocardium. The cytotoxic T-cells response is one of the most important mechanisms responsible for the lysis of virus-infected cells as well as for far reaching damage of myocardium. In addition, autoimmune reactions are also observed, when the cytotoxic lymphocytes attack healthy part of the myocardium because of the molecular mimicry. It happens because of the virus-induced cytolysis, when specific cellular antigens from necrotic myocytes such as betal-adrenergic receptors, myosin or M2 muscarinic receptors are released (Pankuweit and Klingel, 2013). In a murine model, it was demonstrated 


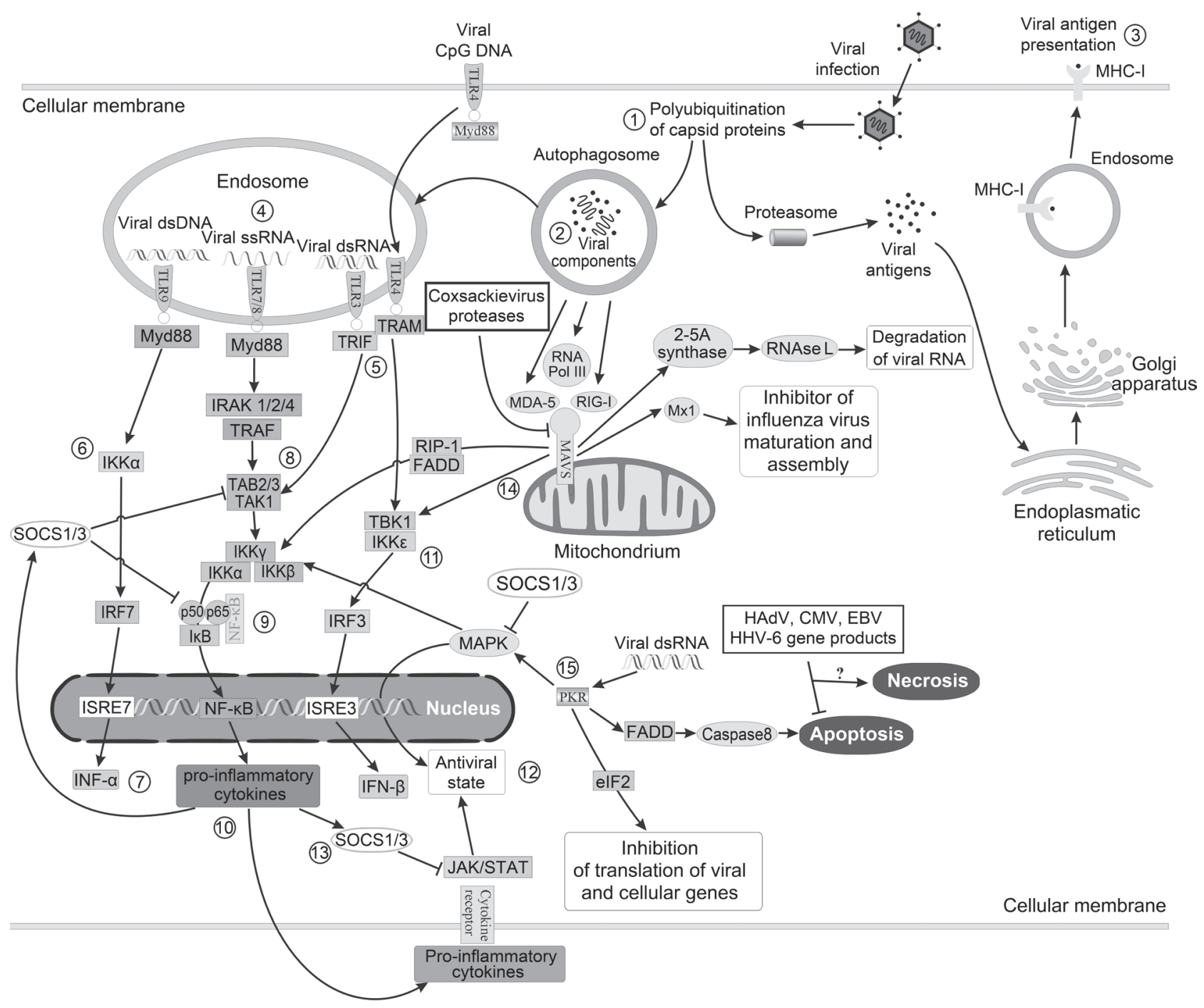

Fig. 1. Major mechanisms activated during viral infection of heart tissue cells.

Directly after viral infection, but also during the entire process of viral replication, viral proteins undergo polyubiquitylation and degradation (1), which leads to trapping of viral components within autophagosomes (2), but also to processing of viral antigens in endoplasmatic reticulum and Golgi apparatus, which, in turn, results in presenting of viral antigens to immune cells in MHC-I context (3). After fusion of autophagosome with endosome, viral DNAs and RNAs are recognized by TLRs (4), from which TLRs 3, 4, 7, 8 and 9 are known to react to viral components. The signal from TLRs, activated by their ligands, is transduced further by Myd88 in case of TLRs 7, 8, 9 and cellular membrane-bound TLR4, or by TRIF (5) in case of TLR3, associated with endosomal TLR4. Signal from TLR9/Myd88 leads to activation of IKKa kinase (6), which phosphorylates IRF7 which results eventually in interferon alpha synthesis (7), while Myd88-transduced signals from TLRs 7 and 8 activate NF- $\mathrm{KB}$ pathway via IRAK/TRAF-TAB/TAK route (8), where TAK-1 kinase phosphorylates IKK $\alpha / \beta / \gamma$ complex, resulting in deactivation of I $\mathrm{B}$, an inhibitor which retains NF- $\kappa \mathrm{B}$ in $c y t$ toplasm (9). Released NF- $\mathrm{KB}$ migrates to the nucleus, where it initiates expression of over 150 genes, including proinflammatory cytokines and proapoptotic genes (10). NF- $\mathrm{kB}$ pathway is also activated by signal from TLR3/TRIF, while interferon beta is expressed in reaction to a signal coming from endosomal TLR4, transduced by TBK/IKKe kinases (11). Autocrine reaction to both type-I interferons and proinflammatory cytokines via JAK-STAT results in expression of numerous genes, initiating antiviral state within a cell (12), and this process is negatively controlled by SOCS proteins (13), synthetized in reaction to proinflammatory cytokines. Stimulated by viral components, also MAVS-associated RLRs (14) induce signal cascade involving TBK1and IKKa/ $\beta / \gamma$-depending pathways. Versatile inducer of antiviral response, PKR (15), initiates antiviral state of the cell (via MAPK), activates eIF2 and apoptotic process (via caspase 8).

that additional, host-depending genetic factors increase the risk of the autoimmune reactions. It is considered that direct viral injury, cytokine context and level of proinflammatory immune reaction together are responsible for determining the severity of MC and probability of drift from acute to subacute stage (Yajima, 2011). The activation of the acquired immunity results in chronic inflammatory response in myocardium and may lead to organ dysfunction due to fibrosis and remodeling of the heart muscle. It can also cause damage due to necrosis and switching on the autophagy of the cardiomyocytes. Recently, it was found that the ubiquitin-proteasome system and lysosome pathways are one of the main factors of the viral infectivity and its inhibition reduces CVB replication in murine (Luo et al., 2003). Fairweather and Rose (2007) showed, that viral genome can be detected in heart tissue during chronic myocarditis. Mechanism of long-term coxsackievirus persistence 
in the presence of an intact immune system remains unclear (Yajima, 2011). Patient may present dyspnoea, chest pain, heart palpitations, decreased exercise tolerance, increased sweating and fainting.

Myopathy phase is the next stage of an infection, where generally it is impossible to detect the virus in myocardium. In case of the persistent inflammatory response, the heart may develop iDCM due to the remodeling. Pathogenic role may be played by the antibodies against sarcolemma, myolemma, beta-receptor, acetylcholine receptor, laminin and cardiac conducting tissue. However, the participation of the antibodies against fibrils, stress proteins and intermediate filaments is not entirely clear (Maisch and Pankuweit, 2013). Inflammation may also be followed by release of cytokines (e.g. transforming growth factor) and activation of matrix metalloproteinases (e.g. urokinase-type plasminogen activator), which predispose to fibrosis through the mothers against decapentaplegic homolog (SMAD) signaling cascade and cardiac dilation (Sagar et al., 2012). Matrix metalloproteinases (MMPs) which can degrade the different components of the cardiac tissue are overexpressed during viral MC and may contribute to tissue remodeling (Li et al., 2009). Additional mechanisms responsible for post-inflammatory remodeling of the heart tissue include enhanced fibrosis as the effect of osteopontin and matricellular protein Cyr61 activity (Pankuweit and Klingel, 2013). At this step, we can observe systolic dysfunction of the left ventricule and walls hypokinesis, often correlating with ECG disorders, especially the ST-T changes. Right ventricular dysfunction is less widespread. Formation of thrombi within the heart cavities and pericardial effusion also can occur.

Furthermore, the presence of the virus, or its components, in cardiac muscle without concomitant local immune response at detectable level, as determined in standard histopathologic examination, can also elicit fibrosis, hypertrophy and degeneration of cardiomyocytes observed in iDCM (Kawai and Matsumori, 2013). Pathological changes in heart muscle can provoke chest pain, tachycardia, irregular heart beating, dyspnea at rest and with effort, fatigue, swelling lower limbs, fainting and hyperhidrosis.

Recently, it has been observed that B19V plays increasing role in the induction of MC in Europe and in the US. Acute infection of B19V is typical for childhood, usually manifests as fifth disease and it is infrequently diagnosed in adults (Bultmann et al., 2003). Erythroid progenitor cells are the main site of $\mathrm{B} 19 \mathrm{~V}$ replication, however other cell types can be also susceptible for infection. High prevalence of B19V in the hearts of patients with MC has been reported in repeated observations, which strongly suggests connection of presence of the virus with the induction of inflammation. This process is most probably associated with the impact of B19V on the endothelium (Bock et al., 2010). B19V, as an erythrovirus, utilizes P-receptor and coreceptors (integrins, KU80) on the endothelial cells. After the connection with the receptor, virus may enter the cells and pass to the state of persistent infection in the endothelium of various organs, including heart, and in consequence, B19V can be found in cells of venules, small arteries or arterioles of children and adults (Bultmann et al., 2003). Our knowledge about the pathogenetic mechanisms of MC induction by B19V is limited due to the lack of appropriate animal model. Despite this problem, few years ago the first results utilizing murine model for the investigation of B19V recombinant antigen impact on myocardium were published (Pankuweit and Klingel, 2013), revealing that mice treated with a recombinant VP1 protein of the B19V developed myocardial injury. Damage was accompanied by the increasing level of aspartate aminotransferase (AST), lactate dehydrogenase (LDH) and creatine kinase isoenzyme (CK-MB) in serum (Nie et al., 2010). The second study showed, that mice which received antibodies against B19V VP1 unique region, developed cardiac injury due to inflammation process (Tzang et al., 2011). Treatment by IFN- $\alpha$ or IFN- $\beta$ almost does not affect to the presence of the $\mathrm{B} 19 \mathrm{~V}$ in the cells, however cell analyses of infected immortalized human microvascular endothelial cells (HMEC-1) proved, that external administration of IFN- $\beta$ can obstruct B19V reactivation and improves endothelial cells viability (Schmidt-Lucke et al., 2010).

Summarizing, both viral infection and subsequent reaction of the immune system causes destruction of the heart tissues (Fig. 2). The damage may be a result of direct viral cytopathic effect, inflammation, necrosis and autophagy. We can divide cardiomyotropic viruses into two main groups. First group comprises viruses for which cardiomyocytes are the most important kind of targeted cells (enteroviruses and adenoviruses). Viruses from this group demonstrate strong cytopathic effect. Enteroviruses usually exhibit low risk of persistence, but in some cases their RNA remains detectable in myocardial cells long time after the acute infection. Adenoviruses are very poorly investigated in terms of the pathogenesis of heart infections. Moreover, this picture is also complicated by their long-term presence in lymphoadenoidal tissue, and potential role of this reservoir as a constant source of low-level adenoviral viremia. The viruses from second group, betaherpesviruses, including CMV and HHV-6, infect endothelium and cardiomyocytes, while DNA or antigens of B19V is found, first of all, in endothelial cells. There is a very limited number of data indicating the presence of the B19V DNA in cardiomyocytes of adults and foetuses (O'Malley et al., 2003). Thus, this problem is still open 


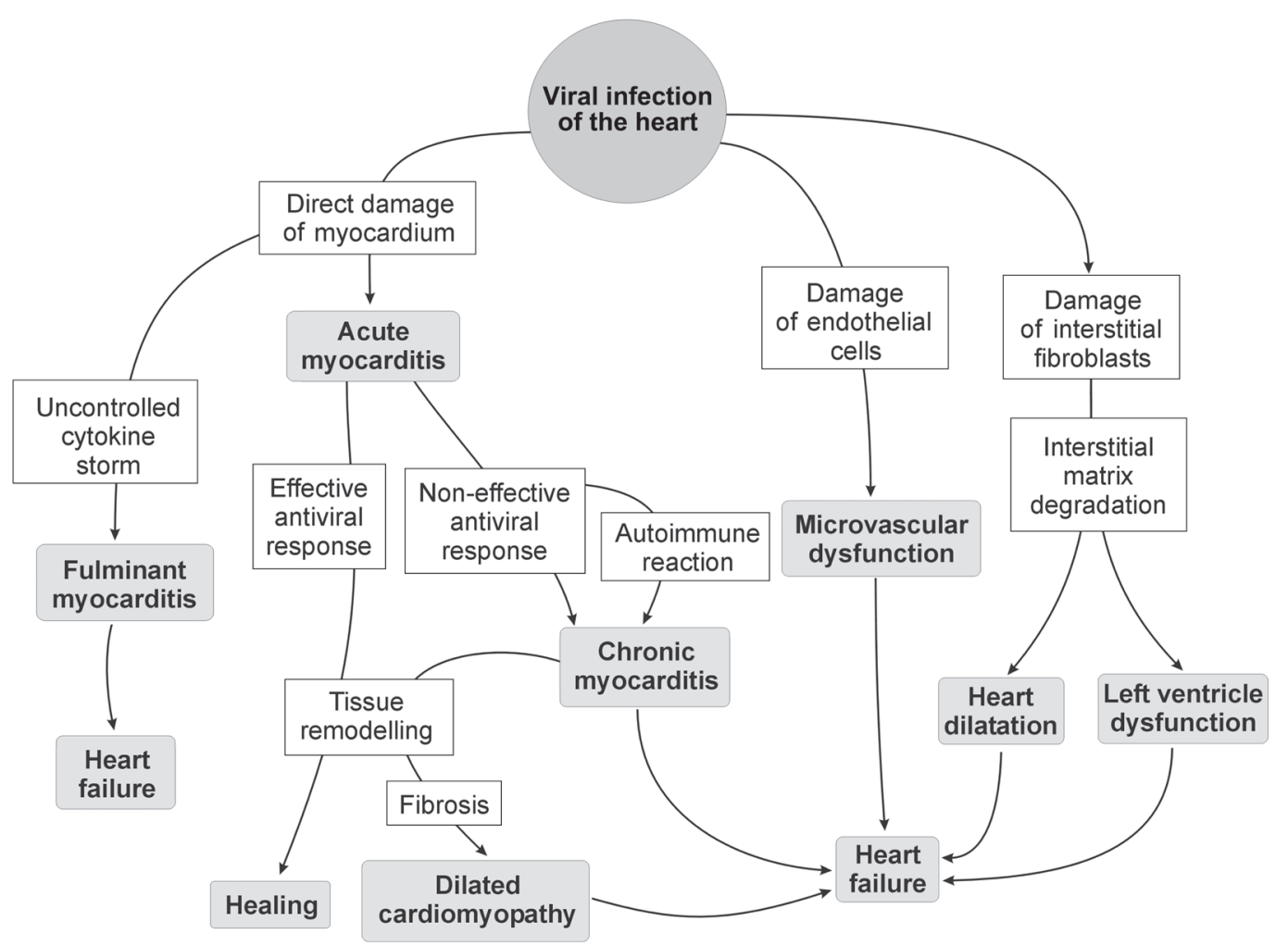

Fig. 2. Potential effects of viral infection of the heart.

Infection of cardiomyocytes, caused by enteroviruses, may result in direct cytopathic effect, resulting in cell damage or death. Prompt activation of intracellular antiviral mechanisms and both innate and acquired immune mechanisms should result in strong local immune response and elimination of both viral particles and infected cells (acute myocarditis). Rarely observed fulminant myocarditis is caused usually by disturbances in control mechanisms of inflammation. Errors in antiviral response, e.g. ineffective or delayed innate immune response, weak cytotoxic T-cell response or insufficient antibody production allows the virus to multiply and spread within the heart. Prolonged presence of the virus in cardial tissues may result in constant, but still ineffective infiltration of immune cells, and may lead to chronic inflammation. Further, this dynamic process, accompanied by constant loss of damaged contractile tissue in appearance of fibrosis, may result in dilated cardiomyopathy, and often ends in progressive heart failure, despite introduced treatment. Damage resulting from infection of interstitial tissue, caused first of all by parvoviruses and herpesviruses may result in heart dilatation and left ventricle dysfunction. These viruses are also known to infect the endothelium of local blood vessels, which may result in worsening of clinical picture because of microvascular dysfunction.

to debate, including the question about the ability of B19V to replicate actively in adult cardiomyocyte.

In terms of virological diagnostics, accurate identification of etiologic agent of viral MC or iDCM is one of the most difficult tasks set, and efforts to establish effective diagnostic procedures have been making since late forties of past century (Woodruff, 1980). Virological laboratory diagnosis, including direct methods, specifically virus isolation in cell lines and electron microscopy, considered substantially as more reliable than serological examinations. Despite the unambiguous answer provided in case of isolation of the virus from EMB sample, the low recovery rate of the virus was the main limitation of isolation method. It results in some percentage of false-negative outcomes, very hard to assess without the reference method. Low amount of material obtained during biopsy, sampling inadequacies and technical problems with isolation of the virus from tissues resulted in a weak usefulness of this method, especially in the late course of heart infection (Martino et al., 1994).
The biopsies were initially evaluated only histologically according to the Dallas criteria (Aretz, 1987). The presence of an inflammatory infiltrate with or without necrosis on conventionally stained heart-tissue sections evaluated under light microscopy is required for the histological diagnosis of myocarditis. These criteria are limited by variability in interpretation (39\% among pathologists examining the same cardiac tissue samples), lack of prognostic value, and low sensitivity (the Dallas criteria were absent in $50 \%$ of the specimens containing PCR-proven viral pathogens), in part due to sampling error (Martin et al., 1994; Mason et al., 1995; Baughman, 2006). For this reason, the solitary use of the Dallas criteria to diagnose myocarditis is poor. To address the shortcomings, in 1999, the WHF and ISFC updated the conventional histological criteria for diagnosis of myocarditis by the introduction of immunohistochemical methods (staining surface antigen, such as anti-CD3, anti-CD4, anti-CD68 and anti-human leukocyte antigens). Criteria for immunohistological diagnosis of myocarditis are specified quantitatively as 
14 infiltrating leukocytes $/ \mathrm{mm}^{2}$, preferably T-lymphocytes (e.g. CD3) or activated T-cells (CD45ro) (Maisch et al., 1999). Criteria that are based on immunoperoxidase staining, have greater sensitivity and may have a prognostic value. However, many observations confirm, that routine histological and immunohistological analysis is too insensitive to detect myocardial inflammation accurately in acute phase of disease, as well as in chronic phase. Finally, even if conventional histological and immunohistological analysis allow to diagnose inflammation in heart, they do not allow to identify virus and in consequence to use of targeted treatment.

Problems with detection of the viral pathogen in cases of suspected MC or iDCM with use of virus isolation techniques led to searches for alternative diagnostic methods. Before the popularization of molecular biology and methods of direct detection of viral antigens in situ, diagnosis was often established on the basis of detection of specific antibodies directed against cardiotropic viruses in patients' sera. Serological investigations utilized paired sera examination, detection of IgM-class antibodies or clinical seroepidemiological studies (Leslie et al., 1989). Utility of serological methods was disputed from the very beginning of their application. In 2011 Mahfoud et al. published their observations conducted on the group of 124 patients with symptoms of acute MC. The authors compared results of identification of viral etiologic agent of MC performed with serological (examination of paired sera with fourfold or more antibodies titers increase) and direct methods (detection of viral nucleic acids in EMBs with real-time PCR). It was shown, that the concordance of the results between both methods was $4 \%$, whereas the positive predictive value of serological methods was $9 \%$.

Another diagnostic approach included the isolation of the virus from sites other than EMB (upper respiratory tract, urine or feces) during acute phase of infection in patients with clinical and histopathological changes indicating viral MC (Woodruff, 1980). Diagnostic value of these results in adult patients was not verified with evidence-based medicine standards. However, the wide distribution of viruses with cardiotropic potential in population, along with frequent asymptomatic infections of upper respiratory and gastrointestinal tract, as well as probability of mixed infection with two or more viruses, indicates possibility of isolation of the "bystander" virus instead of pathogen responsible for MC or iDCM.

Contemporary diagnosis leading to identification of etiological agent of viral MC/iDCM relies on direct methods, first of all on detection of viral nucleic acids or antigens in EMB samples, rarely on electron microscopy (Kühl and Schultheiss, 2009). According to the current recommendations, EMB and peripheral blood samples should be investigated, and PCR, realtime PCR (qPCR) and in situ hybridization (ISH) are the methods acknowledged nowadays (Cooper et al., 2007; Basso et al., 2013). In extended investigations conducted during last years, genetic material of viruses was detected in 19-94\% of examined EMB samples. The results revealed rather coherent group of identified viruses. On the other hand the significant differences in detection frequencies of particular viruses were also observed. In the investigations which embraced wide spectrum of viruses sought after, B19V was the most frequent (found in 2.5-60\% of examined EBM samples), followed by HEV (1-33\%), HHV-6 (8-30\%), HAdV (2-23\%), CMV (1-5\%) and EBV (0.5-5\%) with slight differences between results obtained in patients with MC and iDCM (Bowles et al., 2003; Pankuweit et al., 2003; Mahrholdt et al., 2004; Kühl et al., 2005; Caforio et al., 2007; Bock et al., 2010).

Our growing knowledge about the complex mechanisms triggered during viral infections of the heart, their impact on both short- and long-time consequences affecting patient, along with important change in our consciousness of the most prevalent viral agents responsible for acute and chronic heart damage are one of the most important issues in contemporary cardiology. With revealed futility of serological methods and poor predictive value of classical histopathologic criteria, there is growing need for more precise and reliable diagnostic techniques, allowing rapid and unambiguous diagnosis in cases of suspected virus-related heart damage. This gap has been at least partially filled with the advent of modern direct diagnostic methods, based on molecular biology techniques, but still there is more questions than answers. The most urgent issues include introduction of EMB sampling standardization, assessment of the clinical impact of heart tissue viral load during both acute and chronic infection, and identification of macroscale changes in heart tissue, corresponding at the desired level with virus-driven infectious process. There is also growing number of data, suggesting that deficiency in early antiviral innate intracellular immunity may be an important factor responsible for increased risk of development of the chronic heart diseases resulting from viral infections, what indicates new direction in the search for diagnostic methods. In conclusion, depiction of basic processes accompanying viral infection of the heart, both at macro- and microscale should help in the elaboration and introduction of more efficient diagnostic protocols.

\section{Acknowledgments}

Partially financed from National Science Centre grant no. UMO-2014/13/B/N24/03832. 


\section{Literature}

Abston E.D., M.J. Coronado, A. Bucek, D. Bedja, J. Shin, J.B. Kim, E. Kim, K.L. Gabrielson, D. Georgakopoulos, W. Mitzner and others. 2012. Th2 regulation of viral myocarditis in mice: different roles for TLR3 vs. TRIF in progression to chronic disease. Clin. Dev Immunol. 2012: 129486.

Aretz H.T. 1987. Myocarditis: the Dallas criteria. Hum. Pathol. 18: 619-624.

Blauwet L.A. and L.T. Cooper. 2010. Myocarditis. Prog. Cardiovasc. Dis. 52(4): 274-88.

Basso C., F. Calabrese, A. Angelini, E. Carturan and G. Thiene. 2013. Classification and histological, immunohistochemical, and molecular diagnosis of inflammatory myocardial disease. Heart Fail. Rev. 18(6): 673-681.

Baughman K.L. 2006. Diagnosis of myocarditis: death of Dallas criteria. Circullation. 113: 593-595.

Bergelson J.M., J.A. Cunningham, G. Droguett, E.A. Kurt-Jones, A. Krithivas, J.S. Hong, M.S. Horwitz, R.L. Crowell and R.W. Finberg. 1997. Isolation of a common receptor for Coxsackie B viruses and adenoviruses 2 and 5. Science 275: 1320-1323.

Bock C.T., K. Klingel and R. Kandolf. 2010. Human parvovirus B19-associated myocarditis. N. Engl. J. Med. 362(13): 1248-1249.

Bowles N.E., J. Ni, D.L. Kearney, M. Pauschinger, H.P. Schultheiss, R. McCarthy, J. Hare, J.T. Bricker, K.R. Bowles and J.A. Towbin. 2003. Detection of viruses in myocardial tissues by polymerase chain reaction. evidence of adenovirus as a common cause of myocarditis in children and adults. J. Am. Coll. Cardiol. 42(3): 466-472.

Bültmann B.D., K. Klingel, K. Sotlar, C.T. Bock, H.A. Baba M. Sauter and R. Kandolf. 2003. Fatal parvovirus B19-associated myocarditis clinically mimicking ischemic heart disease: an endothelial cell-mediated disease. Hum. Pathol. 34: 92-95.

Caforio A.L., F. Calabrese, A. Angelini, F. Tona, A. Vinci, S. Bottaro, A. Ramondo, E. Carturan, S. Iliceto, G. Thiene and others. 2007. A prospective study of biopsy-proven myocarditis prognostic relevance of clinical and aetiopathogenetic features at diagnosis. Eur. Heart J. 28(11): 1326-1333.

Cooper L.T., K.L. Baughman, A.M. Feldman, A. Frustaci, M. Jessup, U. Kühl, G.N. Levine, J. Narula, R.C. Starling, J. Towbin and others. 2007. The role of endomyocardial biopsy in the management of cardiovascular disease: a scientific statement from the American Heart Association, the American College of Cardiology, and the European Society of Cardiology. Endorsed by the Heart Failure Society of America and the Heart Failure Association of the European Society of Cardiology. J. Am. Coll. Cardiol. 50(19): 1914-1931.

Dennert R., H.J. Crijns and S. Heymans. 2008. Acute viral myocarditis. Eur. Heart. J. 29(17): 2073-2082.

Fairweather D., S. Frisancho-Kiss, S.A. Yusung, M.A. Barrett, S.E. Davis, S.J. Gatewood, D.B. Njoku and N.R. Rose. 2004. IFN-g protects against chronic viral myocarditis by reducing mast cell degranulation, fibrosis, and the profibrotic cytokines TGF-b1, IL-1b, and IL-4 in the heart. Am. J. Pathol. 165: 1883-1894.

Fairweather D. and N. Rose. 2007. Coxsackievirus-induced myocarditis in mice: A model of autoimmune disease for studying immunotoxicity. Methods. 41: 118-122.

Fuse K., G. Chan, Y. Liu, P. Gudgeon, M. Husain, M. Chen, W.C. Yeh, S. Akira and P.P. Liu. 2005. Myeloid differentiation factor- 88 plays a crucial role in the pathogenesis of Coxsackievirus B3-induced myocarditis and influences type I interferon production. Circulation. 112: 2276-2285.

Herzum M., V. Ruppert, B. Kuytz, H. Jomaa, I. Nakamura and B. Maisch. 1994. Coxsackievirus B3 infection leads to cell death of cardiac myocytes. J. Mol. Cell Cardiol. 26(7): 907-913.
Huber S.A., D. Sartini and M. Exley. 2002. Vgamma4(+) T cells promote autoimmune $\mathrm{CD} 8(+)$ cytolytic T-lymphocyte activation in coxsackievirus B3-induced myocarditis in mice: role for CD4(+) Th1 cells. J. Virol. 76: 10785-10790.

Kawai C. 1999. From myocarditis to cardiomyopathy: mechanisms of inflammation and cell death: learning from the past for the future. Circulation. 99: 1091-100.

Kawai C. and A. Matsumori. 2013. Dilated cardiomyopathy update: infectious-immune theory revisited. Heart Fail. Rev. 18(6): 703-714. Kühl U., M. Pauschinger, B. Seeberg, D. Lassner, M. Noutsias, W. Poller and H.P. Schultheiss. 2005. Viral persistence in the myocardium is associated with progressive cardiac dysfunction. Circulation. 112(13): 1965-1970.

Kühl U., M. Pauschinger, P.L. Schwimmbeck, B. Seeberg, C. Lober, M. Noutsias, W. Poller and H.P. Schultheiss. 2003. Interferon-beta treatment eliminates cardiotropic viruses and improves left ventricular function in patients with myocardial persistence of viral genomes and left ventricular dysfunction. Circulation. 107: 2793-2798.

Kühl U. and H.P. Schultheiss. 2009. Viral myocarditis: diagnosis, aetiology and management. Drugs. 69(10): 1287-1302.

Lafyatis R. and A. Farina. 2012. New insights into the mechanisms of innate immune receptor signalling in fibrosis. The Open Rheum. J. 6(Suppl 1: M2): 72-79.

Leslie K., R. Blay, C. Haisch, A. Lodge, A. Weller and S. Huber. 1989. Clinical and experimental aspects of viral myocarditis. Clin. Microbiol. Rev. 2(2): 191-203.

Li K., W. Xu, Q. Guo, Z. Jiang, P. Wang, Y. Yue and S. Xiong. 2009. Differential macrophage polarization in male and female BALB/c mice infected with coxsackievirus B3 defines susceptibility toviral myocarditis. Circ. Res. 105: 353-364.

Luo H., J. Zhang, C. Cheung, A. Suarez, B.M. McManus and D. Yang. 2003. Proteasome inhibition reduces coxsackievirus B3 replication in murine cardiomyocytes. Am. J. Pathol. 163:381-385. Mahfoud F, B. Gärtner, M. Kindermann, C. Ukena, K. Gadomski, K. Klingel, R. Kandolf, M. Böhm and I. Kindermann. 2011. Virus serology in patients with suspected myocarditis: utility or futility? Eur Heart J. 32: 897-903.

Mahrholdt H., C. Goedecke, A. Wagner, G. Meinhardt, A. Athanasiadis, H. Vogelsberg, P. Fritz, K. Klingel, R. Kandolf and U. Sechtem. 2004. Cardiovascular magnetic resonance assessment of human myocarditis: a comparison to histology and molecular pathology. Circulation. 109: 1250-1258.

Maisch B. and S. Pankuweit. 2013. Standard and etiology-directed evidence-based therapies in myocarditis: state of the art and future perspectives. Heart Fail. Rev. 18(6): 761-795.

Maisch B., B.D. Bültmann, S. Factor, H.J. Gröne, G. Hufnagel, K. Kawamura, U. Kühl, E.J. Olsen, S. Pankuweit and R. Virmani. 1999. World Heart Federation con-sensus conferences' definition of inflammatory cardiomyopathy (myocarditis): report from two expert committees on histology and viral cardiomyopathy. Heartbeat. 4: 3-4.

Martin A.B., S. Webber, F.J. Fricker, R. Jaffe, G. Demmler, D. Kearney, Y.H. Zhang, J. Bodurtha, B. Gelb and J. Ni. 1994. Acute myocarditis. Rapid diagnosis by PCR in children. Circulation. 90: 330-339.

Martino T.A., P. Liu and M.J. Sole. 1994. Viral infection and the pathogenesis of dilated cardiomyopathy. Circ. Res. 74: 182-188. Mason J.W., J.B. O'Cornell, A. Herscowitz, N.R. Rose, B.M. McManus, M.E. Bilingham and T.E. Moon. 1995. A Clinical trial of immunosupressive therapy for myocarditis. The Myocarditis Treatment Trial Investigators. N. Engl. J. Med. 333: 269-275.

Negishi H., T. Osawa, K. Ogami, X. Ouyang, S. Sakaguchi, R. Koshiba, H. Yanai, Y. Seko, H. Shitara, K. Bishop and others. 2008. A critical link between Toll-like receptor 3 and type I inter- 
feron signaling pathways in antiviral innate immunity. Proc. Natl. Acad. Sci. USA 105(51): 20446-20451.

Nie X., G. Zhang, D. Xu, X. Sun, Z. Li, X. Li, X. Zhang, F. He and Y. Li. 2010. The VP1-unique region of parvovirus B19 induces myocardial injury in mice. Scand. J. Infect. Dis. 42: 121-128.

O'Malley A., C. Barry-Kinsella, C. Hughes, P. Kelehan, D. Devaney, E. Mooney and J. Gillan. 2003. Parvovirus infects cardiac myocytes in hydrops fetalis. Pediatr. Dev. Pathol. 6: 414-420.

Pankuweit S., R. Moll, U. Baandrup, I. Portig, G. Hufnagel and B. Maisch. 2003. Prevalence of the parvovirus B19 genome in endomyocardial biopsy specimens. Hum. Pathol. 34: 497-503.

Pankuweit S. and K. Klingel. 2013. Viral myocarditis: from experimental models to molecular diagnosis in patients. Heart Fail. Rev. 18(6): 683-702.

Sagar S., P.P. Liu and L.T. Jr. Cooper. 2012. Myocarditis. Lancet 379(9817): 738-747

Shi Y., C. Chen, U. Lisewski, U. Wrackmeyer, M. Radke, D. Westermann, M. Sauter, C. Tschöpe, W. Poller, K. Klingel and others. 2009. Cardiac deletion of the Coxsackievirus-adenovirus receptor abolishes Coxsackievirus B3 infection and prevents myocarditis in vivo. J. Am. Coll. Cardiol. 53: 1219-1226.

Schmidt-Lucke C., F. Spillmann, T. Bock, U. Kühl, S. Van Linthout, H.P. Schultheiss and C. Tschöpe. 2010. Interferon Beta Modulates Endothelial Damage in Patients with Cardiac Persistence of Human Parvovirus B19 Infection. J. Infect. Dis. 201-206
Stewart P.L. and G.L. Nemerow. 2007. Cell integrins: commonly used receptors for diverse viral pathogens. Trends in Microbiology 15(11): 500-507.

Tanaka T., T. Kanda, B.M. McManus, H. Kanai, H. Akiyama, K. Sekiguchi, T. Yokoyama and M. Kurabayashi. 2001. Overexpression of interleukin-6 aggravates viral myocarditis: impaired increase in tumor necrosis factor-a. J. Mol. Cell. Cardiol. 33: 1627-1635. Tzang B.S., T.M. Lin, C.C. Tsai, J.D. Hsu, L.C. Yang and T.C. Hsu. 2011. Increased cardiac injury in NZB/W F1 mice received antibody against human parvovirus B19 VP1 unique region protein. Mol. Immunol. 48: 1518-1524.

Woodruff J.F. 1980. Viral myocarditis. A review. Am. J. Pathol. 101(2): 425-484.

Yajima T. 2011. Viral myocarditis: potential defense mechanisms within the cardiomyocyte against virus infection. Future Microbiol. 6: 551-566.

Yasukawa H., T. Yajima, H. Duplain, M. Iwatate, M. Kido, M. Hoshijima, M.D. Weitzman, T. Nakamura, S. Woodard, D. Xiong and others. 2003. The suppressor of cytokine signaling-1 (SOCS1) is a novel therapeutic target for enterovirus-induced cardiac injury. J. Clin. Invest. 111(4): 469-478.

Yuan J., A.L. Cao, M. Yu, Q.W. Lin, X. Yu, J.H. Zhang, M. Wang, H.P. Guo and Y.H. Liao. 2010. Th17 cells facilitate the humoral immune response in patients with acute viral myocarditis. J. Clin. Immunol. 30: 226-234. 